BULLETIN (New Series) OF THE

AMERICAN MATHEMATICAL SOCIETY

Volume 45, Number 4, October 2008, Pages 595-616

S 0273-0979(08)01213-5

Article electronically published on July 1, 2008

\title{
EXOTIC SPHERES AND CURVATURE
}

\author{
M. JOACHIM AND D. J. WRAITH
}

\begin{abstract}
Since their discovery by Milnor in 1956, exotic spheres have provided a fascinating object of study for geometers. In this article we survey what is known about the curvature of exotic spheres.
\end{abstract}

\section{INTRODUCTION}

Exotic spheres are manifolds which are homeomorphic but not diffeomorphic to a standard sphere. In this introduction our aims are twofold: First, to give a brief account of the discovery of exotic spheres and to make some general remarks about the structure of these objects as smooth manifolds. Second, to outline the basics of curvature for Riemannian manifolds which we will need later on. In subsequent sections, we will explore the interaction between topology and geometry for exotic spheres. We will use the term differentiable to mean differentiable of class $C^{\infty}$, and all diffeomorphisms will be assumed to be smooth.

As every graduate student knows, a smooth manifold is a topological manifold that is equipped with a smooth (differentiable) structure, that is, a smooth maximal atlas. Recall that an atlas is a collection of charts (homeomorphisms from open neighbourhoods in the manifold onto open subsets of some Euclidean space), the domains of which cover the manifold. Where the chart domains overlap, we impose a smooth compatibility condition for the charts doC, chapter 0] if we wish our manifold to be smooth. Such an atlas can then be extended to a maximal smooth atlas by including all possible charts which satisfy the compatibility condition with the original maps. It is far from obvious that there are manifolds which (up to diffeomorphism) admit more than one distinct smooth structure. Indeed the discovery of such exotic smooth structures was a big surprise. It is also not obvious that some topological manifolds admit no smooth structures [HBJ, p. 114] [FQ, 10.2A], though such objects will not concern us in this article.

In dimensions one, two, and three it is well known that every topological manifold admits a unique smooth structure [FQ, 8.3]. The lowest dimension in which exotic smooth structures exist is dimension four. It is perhaps surprising that familiar manifolds such as $\mathbb{R}^{4}$ can admit exotic differentiable structures! Dimension four is somewhat special from the point of view of such structures. Many four-dimensional manifolds (e.g., $\mathbb{R}^{4}$ ) are known to admit infinitely many distinct smooth structures $\mathrm{FQ}, 8.4 \mathrm{C}]$. However, this phenomenon cannot occur in higher dimensions. In dimensions at least five, any manifold has at most a finite number of smooth structures, and in many cases this number is one (see [HM] and [KS]).

Received by the editors May 27, 2008

2000 Mathematics Subject Classification. Primary 53C20.

(C)2008 American Mathematical Society Reverts to public domain 28 years from publication 
Historically, the first manifolds discovered to admit exotic smooth structures were spheres, specifically $S^{7}$. These structures were found by Milnor in 1956 [M1]. We give a brief account of the background of their discovery, following Milnor's own description in M5.

Milnor was studying the topology of high-dimensional manifolds, and in particular manifolds of dimension $2 n$ which are $(n-1)$-connected. This connectedness condition means that the manifold is connected and the homotopy groups $\pi_{1}, \ldots, \pi_{n-1}$ are all zero. It was a reasonable condition to consider as the topology of such a family of manifolds seemed tractable. Narrowing down the focus of study further, Milnor considered 3-connected 8-manifolds $M^{8}$ for which the middle-dimensional cohomology (with integer coefficients) has a single generator. In this situation it is always possible to find an embedded $S^{4} \subset M^{8}$ which generates $H^{4}(M)$. A tubular neighbourhood of this $S^{4}$ is a disc bundle with fibre $D^{4}$, and it can be shown that $M^{8}$ must be the union of this bundle with an 8-disc. It follows that the boundary of the disc bundle must be $S^{7}$, and in particular this means that the bundle cannot be trivial (that is, a product $S^{4} \times D^{4}$ ).

To understand the possibilities for $M^{8}$, it was necessary to investigate the possible $D^{4}$-bundles over $S^{4}$. We can view $S^{4}$ as $D^{4} \cup_{S^{3}} D^{4}$. As $D^{4}$ is contractible, any bundle over $S^{4}$ restricted to either hemisphere is trivial. Therefore, the problem of understanding $D^{4}$-bundles over $S^{4}$ reduces to identifying the ways in which the fibre discs over the boundary of each hemisphere $D^{4}$ can be identified when the two hemispheres are glued. Such an identification is given by a map $S^{3} \rightarrow S O(4)$, and it is not difficult to see that the different possible bundles are in one-to-one correspondence with the elements of $\pi_{3} S O(4) \cong \mathbb{Z} \oplus \mathbb{Z}$. This correspondence can be realised explicitly in the following way. For a pair $(m, n) \in \mathbb{Z} \oplus \mathbb{Z}$, we consider the bundle constructed by using an identification map $f_{(m, n)}: S^{3} \rightarrow S O(4)$ defined by

$$
f_{(m, n)}(u)(v)=u^{m} v u^{n},
$$

where $u$ is a unit quaternion, $v \in \mathbb{R}^{4}$, and quaternion multiplication is to be understood on the right-hand side. A little algebraic topology shows that for the boundary of the disc bundle corresponding to the pair $(m, n)$ to be homotopy equivalent to $S^{7}$, we need $m+n=1$ or $m+n=-1$. Of course, this gives infinitely many choices. Let us assume from now on that $m+n=1$.

For these cases, consider the corresponding $M^{8}$. A compact, oriented $4 n$ dimensional manifold has a signature, which is a number depending on the cup product structure in the middle-dimensional cohomology (see [MS, p. 224] for the definition). For a smooth, compact, oriented 8-manifold, Hirzebruch showed that the signature $\sigma\left(M^{8}\right)$ is given by the formula

$$
\sigma\left(M^{8}\right)=\frac{1}{45}\left(7 p_{2}\left[M^{8}\right]-p_{1}^{2}\left[M^{8}\right]\right)
$$

where $p_{1}^{2}\left[M^{8}\right]$ and $p_{2}\left[M^{8}\right]$ are so-called Pontrjagin numbers of the manifold (see [MS, §16] for the definition of Pontrjagin numbers, and [MS, p. 224] for the general statement of Hirzebruch's signature theorem). Now the signature of $M$ has to be +1 or -1 (a consequence of the dimension of $M$ and Poincaré duality), and after fixing an orientation for $M$ we can assume that the signature is +1 . For our manifolds $M^{8}$ it turns out that $p_{1}^{2}\left[M^{8}\right]=4(m-n)^{2}=4(2 m-1)^{2}$ since $m+n=1$. With this 
in mind, the above equation can be solved for $p_{2}\left[M^{8}\right]$ :

$$
p_{2}[M]=\frac{4(2 m-1)^{2}+45}{7} .
$$

The key point is that $p_{2}[M]$ must be an integer (since we are using cohomology with integer coefficients), but for certain choices of $m$ (for example $m=2$ ) the above formula does not yield an integer! The upshot of this is that there are $D^{4}$-bundles over $S^{4}$ with boundary homotopically equivalent to $S^{7}$, for which it is impossible to add a $D^{8}$ to give a smooth manifold.

Milnor went on to show (using Morse theory) that the boundary of these disc bundles is actually homeomorphic to $S^{7}$. Thus the boundary of such disc bundles is homeomorphic, but cannot be diffeomorphic to $S^{7}$. In other words, this construction yields exotic spheres.

Using surgery theory, Kervaire and Milnor [KM] were able to show how large the family of exotic spheres is in each dimension greater than four. Although in each case this number is finite, there are exotic spheres in infinitely many dimensions, and in dimensions $4 n+3$ the number of exotic spheres grows very rapidly with $n$. The question of whether there is an exotic $S^{4}$, and if so, how many there are is still open today. This is essentially the smooth Poincaré conjecture in dimension four.

Following Milnor's initial discovery, many different topological descriptions of exotic spheres have been found. Taking orientation into account, there are 27 exotic spheres in dimension seven, and ignoring orientation there are 14. It can be shown that 10 out of the 14 can be expressed as $S^{3}$-bundles over $S^{4}$, and these are known as the Milnor spheres. Subsequently, it was shown that many exotic 15spheres can be described as $S^{7}$-bundles over $S^{8}$. In 1966 Brieskorn Bk was able to realise many odd-dimensional exotic spheres as explicit subsets of Euclidean space. We give an explicit description of these manifolds in Section 2 (for dimensions 1 modulo 4) and in Section 3 (for dimensions 3 modulo 4).

A general approach to describing exotic spheres is provided by the twisted sphere construction. A twisted sphere is a manifold constructed by gluing two discs $D^{n}$ along their boundaries using an orientation preserving diffeomorphism $S^{n-1} \rightarrow$ $S^{n-1}$. It is easy to see that such a manifold is homeomorphic to $S^{n}$; however, we can also produce exotic spheres by this process in the case where the diffeomorphism used in the construction is not smoothly isotopic to the identity. In fact, in dimensions other than four, every exotic sphere is a twisted sphere. In dimension four, Cerf [Ce showed that the only twisted sphere is the standard sphere, so if an exotic 4-sphere exists, it cannot be a twisted sphere. The difficulty with this approach is specifying the diffeomorphism $S^{n-1} \rightarrow S^{n-1}$ required to construct a given exotic sphere. Explicit examples of such maps are hard to find: see for instance [ $\mathrm{Du}$, where an explicit diffeomorphism $S^{6} \rightarrow S^{6}$ is described which yields an exotic 7 -sphere in the twisted sphere construction.

Although these and other constructions have proved illuminating and useful in many cases, it is important to note that there are exotic spheres for which no explicit construction is known. We will say a little more about this is Section 3.

The term homotopy sphere is often used when discussing exotic spheres, and it is important to define this. A homotopy sphere $\Sigma^{n}$ is a smooth manifold with the same homotopy type as $S^{n}$. Thus every exotic sphere is a homotopy sphere. Of course any standard sphere is trivially a homotopy sphere. In dimensions at least 
five, any smooth manifold with the homotopy type of a sphere must be homeomorphic to a sphere. This is the Generalised Poincaré Conjecture, proved by Smale in Sm1. Thus in these dimensions the set of diffeomorphism classes of homotopy spheres is precisely the union of the diffeomorphism class of the standard sphere with the diffeomorphism classes of the exotic spheres. In dimensions one and two, homotopy spheres are always diffeomorphic to the standard spheres. In dimension three, it has been known for many years that the differentiable structure on $S^{3}$ is unique; in other words there is no exotic $S^{3}$. Moreover every 3-manifold has a unique differentiable structure, and any two homeomorphic 3-manifolds are necessarily diffeomorphic $[\mathrm{FQ}, 8.3]$. On the other hand, it was not known if a homotopy $S^{3}$ was necessarily homeomorphic (and therefore diffeomorphic) to $S^{3}$. This is the famous Poincaré conjecture, which has recently been resolved in the affirmative by Perelman ([P1], [P2], [P3]). Thus any homotopy 3 -sphere is diffeomorphic to $S^{3}$. In dimension four, Freedman [Fr] showed that any homotopy 4-sphere is homeomorphic to $S^{4}$; however as indicated above, the question of whether such a manifold is necessarily diffeomorphic to $S^{4}$ still remains mysterious. Note that it is often more convenient to deal with homotopy spheres (as opposed to exotic spheres) as the set of diffeomorphism classes of oriented homotopy spheres in any given dimension (with the possible exception of dimension four) has an algebraic structure: it is a finite abelian group under the connected sum operation, with the standard sphere playing the role of the identity element. For example, Kervaire and Milnor $[\mathrm{KM}$ identify the number of exotic spheres in each dimension (except four) by determining the order of this group. In actual fact, Kervaire and Milnor consider the group of $h$-cobordism classes of homotopy spheres, as opposed to the group of diffeomorphism classes. However, it follows from Smale's $h$-cobordism theorem $\mathrm{Sm} 2$ that in dimensions greater than four, two homotopy spheres are $h$-cobordant if and only if they are diffeomorphic. As mentioned, in dimension three this follows from Perelman's work. In dimension four it is shown in [KM] that the group of $h$-cobordism classes of homotopy spheres is trivial, but of course the possible differentiable structures are unknown.

We now turn our attention briefly to Riemannian geometry, and in particular to the notion of curvature. Any smooth manifold can be equipped with a smooth Riemannian metric, that is, a smoothly varying inner product on each tangent space. A Riemannian metric allows us to do geometry on the manifold. In particular, a Riemannian metric endows the manifold with shape, and, therefore, it makes sense to try and quantify the curvature of such an object. Any smooth manifold will admit a huge family of Riemannian metrics. The metrics in this family will display varying curvature characteristics. Of interest to geometers are manifolds that admit metrics satisfying some special curvature condition. The 'special conditions' on which this article focuses require the curvature to have a particular sign.

The most widely known measure of curvature is the Gaussian curvature in dimension two. This is a (smooth) real-valued function on the manifold. Heuristically, if the Gaussian curvature at a point is positive, then the geometry in a neighbourhood of the point resembles that of a sphere; if the Gaussian curvature is zero, the local geometry is that of a Euclidean plane, and if the Gaussian curvature is negative, the local geometry resembles that of a saddle. A more concrete interpretation of the sign can be given in terms of triangles. In a surface of positive Gaussian curvature, the angles of a geodesic triangle sum to more than $\pi$; in a surface where the 
Gaussian curvature vanishes, the angles sum to exactly $\pi$; and in the case where the Gaussian curvature is negative, the angles of such a triangle sum to less than $\pi$.

It is not surprising that the possible geometries of a surface are deeply linked to the surface's topology. The Unformisation Theorem for surfaces says that every closed compact surface admits a metric with constant Gaussian curvature. This, combined with the Gauss-Bonnet Theorem (applied to the oriented double cover if the surface is not orientable) shows that the only closed compact surfaces admitting a metric of strictly positive Gaussian curvature are the 2-sphere and the real projective plane. The only closed compact surfaces admitting a metric of identically zero Gaussian curvature are the torus and Klein bottle. The remaining closed compact surfaces are precisely those admitting a metric of strictly negative Gaussian curvature. However, the simplicity of this topology-curvature relationship in dimension two is not mirrored in higher dimensions.

The first geometric issue to deal with in higher dimensions is that there are three common, competing ways in which the curvature can be measured. First, there is a natural generalisation of the notion of Gaussian curvature. Consider a point $p$ in a Riemannian manifold $M^{n}, n \geq 2$. Choose a two-dimensional subspace $S$ in the tangent space $T_{p} M$. Locally, we can find an embedded surface in $M$ containing $p$, which is tangent to $S$ at $p$. (We find such a surface by projecting $S$ onto $M$ using the exponential map at $p$.) Restricting the Riemannian metric to this surface, we can compute a Gaussian curvature. This number is the so-called sectional curvature of $M$ at $p$ corresponding to the tangent plane $S$ doC, p. 132]. The sectional curvature is therefore a real-valued function on the set of tangent 2-planes to $M$.

The other measures of curvature are obtained from the sectional curvature by averaging processes. The next curvature to be introduced is the Ricci curvature. Given a unit tangent vector $u$, extend $u$ to an orthonormal frame $\left\{u, e_{1}, e_{2}, \ldots, e_{n-1}\right\}$ for the tangent space. The Ricci curvature of $u$ is defined to be the sum of the sectional curvatures of the planes spanned by $u$ and $e_{i}$, for $i$ between 1 and $n-1$. The resulting value is independent of the way in which $u$ is extended to a frame. For non-unit vectors, the Ricci curvature varies quadratically with the norm, that is $\operatorname{Ric}(\lambda u)=\lambda^{2} \operatorname{Ric}(u)$. This algebraic property is a consequence of the more usual definition of the Ricci curvature in terms of the Riemann curvature tensor. Thus the Ricci curvature is a smoothly varying real-valued quadratic form on the tangent bundle.

The scalar curvature at a point is simply the sum of Ricci curvatures over any orthonoral frame of tangent vectors at that point. Like the Ricci curvature, the scalar curvature is independent of the choice of frame. The scalar curvature is therefore a smooth real-valued function on the manifold. Clearly, in the process of averaging, we would expect to lose information. Thus the scalar curvature is a weaker notion of curvature than the Ricci curvature, and in turn, the Ricci curvature is a weaker notion than the sectional curvature. (See $[\mathrm{P}, \S 2.2]$ for a detailed introduction to these notions of curvature.)

These higher-dimensional measures of curvature are more difficult to interpret than the Gaussian curvature in dimension two. Roughly speaking, the sectional curvature controls distance (see for example the Toponogov Triangle Comparison Theorem [P, §11.2]). On the other hand, the Ricci curvature is closely related to volume: in particular a lower bound on the Ricci curvature leads to an upper bound 
on the volume of (geodesic) balls of any given radius in the manifold [P, §9.1]. This means, for example, that a unit radius ball in a manifold of positive Ricci curvature has a smaller volume than a unit ball in the Euclidean space of the same dimension. Note that a manifold for which the Ricci curvatures are positive and bounded away from zero must be compact, by the Myers Theorem $[\mathrm{My}$. These results are no longer true if we replace the Ricci curvature by the scalar curvature. The scalar curvature controls volume only locally (compare $[\mathrm{B}, 0.60]$ ), but existence of a positive scalar curvature metric on a manifold also does have topological implications (see for example [St]); however, these are quite weak. For instance, if we form the product of a compact positive scalar curvature manifold with any other compact manifold, this product will also admit a positive scalar curvature metric. As will become clear in subsequent sections, when studying the curvature of exotic spheres, we will be interested primarily in positive (or at least non-negative) curvature.

A major problem in Riemannian geometry is to understand the interrelationship of curvature and topology. Since homotopy spheres have a very straightforward topology (as opposed to smooth topology!) it seems reasonable to focus on this family in order to explore this relationship. The standard (round) sphere is the classic example of a manifold with positive curvature: the $n$-dimensional sphere of radius $R$ has constant sectional curvature $1 / R^{2}$, Ricci curvature $(n-1) / R^{2}$, and scalar curvature $n(n-1) / R^{2}$. By studying the curvature of exotic spheres, we gain some insight into the extent to which the geometry of these objects resembles that of the standard sphere. As we will see, in some cases the geometry is very different indeed.

The following sections focus on the sectional curvature, the Ricci curvature, and scalar curvature, respectively. There are many different topological descriptions of exotic spheres. These different descriptions suggest different approaches to constructing metrics. In turn this is (at least partly) responsible for the diversity of results described in this article. We have attempted to give as comprehensive a picture as possible, and as a consequence in many places have kept details to a minimum. It is hoped that the interested reader will use the many references to develop a deeper understanding of the results described.

Note that throughout this article, all Riemannian metrics are assumed to be complete.

The authors would like to thank Charles Boyer, Jost Eschenburg, Hansjörg Geiges, Thomas Püttmann, Stephan Stolz, Stefan Bechtluft-Sachs, and Burkhard Wilking for reading the preliminary versions of this article, and for their very helpful comments and suggestions.

\section{Sectional Curvature}

We begin our discussion of exotic spheres and the sectional curvature by recalling the Hadamard-Cartan Theorem [Ch, p. 174]. This states that the exponential map

$$
\exp : T_{p} M \rightarrow M
$$

based at any point $p$ in a Riemannian manifold $M^{n}$ with non-positive sectional curvature has maximal rank everywhere in $T_{p} M$. It follows that exp is a covering map, and that $T_{p} M \cong \mathbb{R}^{n}$ is the universal covering space. As an immediate corollary, we see that no exotic sphere can admit a metric of non-positive sectional curvature. Therefore, we turn our attention to metrics of non-negative and positive sectional curvature. 
The following question is fundamental 1

Question. Does at least one exotic sphere support a metric with everywhere (strictly) positive sectional curvature?

If we replace 'positive' with 'non-negative' in the above question, the answer is yes. In [GM], Gromoll and Meyer produced the first example of an exotic sphere with a metric of non-negative sectional curvature. For a long time this was the only known example. Indeed at the time of writing, only a handful of other examples are known. We will now discuss the Gromoll-Meyer sphere and subsequent developments in some detail.

The Gromoll-Meyer sphere is a seven-dimensional exotic sphere. Recall from the Introduction that the diffeomorphism classes of oriented homotopy spheres form a finite abelian group in any given dimension (with the possible exception of dimension four) under the connected sum operation. For details of the structure and order of these groups, see [KM]. Those homotopy spheres of dimension $n$ which bound a parallelisable manifold form a cyclic subgroup denoted $b P_{n+1}$. In dimension seven, the group of oriented homotopy spheres is cyclic of order 28 , and the subgroup bounding parallelisable manifolds is in fact the whole group.

The Gromoll-Meyer sphere is a generator of the group of homotopy spheres in dimension seven. It is realised as a biquotient of Lie groups. Let $S p(n)$ denote the group of $(n \times n)$-matrices with quaternion entries satisfying $A A^{\star}=A^{\star} A=\mathbb{I}_{n}$, where $A^{\star}$ denotes the transpose of the quaternion conjugate of $A$.

We have an action of $S^{3} \times S^{3} \cong S p(1) \times S p(1)$ on $S p(2)$ given by

$$
\left(q_{1}, q_{2}\right) A=\left(\begin{array}{cc}
q_{1} & 0 \\
0 & q_{1}
\end{array}\right) A\left(\begin{array}{cc}
q_{2}^{\star} & 0 \\
0 & 1
\end{array}\right),
$$

where $q_{2}^{\star}$ is the quaternion-conjugate of $q_{2}$. This action is free, and hence restricting the action to the diagonal $D$ in $S p(1) \times S p(1)$ also gives a free action. The GromollMeyer sphere is the quotient manifold $S p(2) / D$.

As $S p(2)$ is a compact Lie group, it admits a bi-invariant metric with nonnegative sectional curvature (see [M2, §21]). The quotient of $S p(2)$ by the free action of $D$ has a unique metric making the projection

$$
\pi: S p(2) \rightarrow S p(2) / D
$$

into a Riemannian submersion. For the theory of Riemannian submersions, see [B; Ch9]. As a consequence of the O'Neill curvature formulas for Riemannian submersions ([ON] and $[\mathrm{B} ; 9.29])$, we see that the submersion metric on the quotient space also has non-negative sectional curvature.

It is interesting to note that almost all known compact Riemannian manifolds of non-negative sectional curvature are biquotients with metrics obtained in a similar fashion to the above. In fact, all known examples of compact simply connected manifolds with (strictly) positive sectional curvature are biquotients. It was shown in [To] and independently in [KZ that the Gromoll-Meyer sphere is the only exotic sphere that can be expressed as a biquotient. For definitions and a general discussion of biquotients, E2 is a basic reference. Subsequent contributions to the theory of biquotients include, for example, [E4] and [Si] as well as [KZ] and [To].

\footnotetext{
${ }^{1}$ On May 6, 2008, P. Petersen and F. Wilhelm announced [PW] that they have constructed a positive sectional curvature metric on the Gromoll-Meyer sphere.
} 
Topologically, the Gromoll-Meyer sphere is a Milnor sphere, that is, an $S^{3}$ bundle with base $S^{4}$. The fibre is $S p(1) \times S p(1) / D$, which is easily identifiable with $S^{3}$. The base of the bundle is $S p(2) /(S p(1) \times S p(1))$. It is possible to give an explicit diffeomorphism between this quotient and $S^{4}$ (see [GM]).

As noted in Section 1 , the $S^{3}$-bundles over $S^{4}$ are classified by $\pi_{3}(S O(4)) \cong \mathbb{Z} \oplus \mathbb{Z}$ via an explicit correspondence. Under this correspondence, the Gromoll-Meyer sphere is associated to the pair $(2,-1)$.

The metric on the Gromoll-Meyer sphere admits an effective isometric action from $O(2) \times S O(3)$. It has strictly positive sectional curvature for all planes in a neighbourhood of $\pi(e)$, where $e \in S p(2)$ is the identity element. However, it can also be shown that for other planes, the sectional curvature is zero. A natural question arising is whether there exists a metric close to the Gromoll-Meyer metric in some sense, but with everywhere positive sectional curvature.

In Wi2, Wilhelm studied the Gromoll-Meyer metric and deformations of the Gromoll-Meyer metric in some detail. Gromoll and Meyer had claimed that their metric had positive sectional curvature almost everywhere. They did not justify this claim, and Wilhelm showed it to be false. In fact their metric has zero curvatures on an open set of points. However, Wilhelm shows that their metric can be deformed to one with positive curvature almost everywhere, and that it admits an effective isometric $S O(3)$ action. He also shows that there is no further smooth deformation of his modified Gromoll-Meyer metric that has positive sectional curvature to first order. This then casts a doubt as to whether it is possible to perturb the metric to one with positive sectional curvature everywhere.

Subsequently, Eschenburg [E5] described a family of metrics on the GromollMeyer sphere, apparently different from the Wilhelm metric, which all have positive sectional curvature almost everywhere. (The paper [E5] contains an omission which was later fixed in [EK]). The original claim about the existence of such metrics was actually made in E2], though without proof. The approach is to consider biinvariant metrics on $S p(2) \times K$, where $K=S p(1) \times S p(1)$, and then to quotient by the diagonal action of $K$. The resulting space is diffeomorphic to $S p(2)$, and the resulting metric is normal homogeneous (and therefore has non-negative sectional curvature). For an appropriate choice of bi-invariant metric on $S p(2) \times K$, the normal homogeneous metric will induce a well-defined metric on the Gromoll-Meyer sphere $S p(2) / D$. By the O'Neill formulas for Riemannian submersions [B, 9.29], this resulting metric will also have non-negative sectional curvature. Eschenburg shows that the points possessing zero curvature planes essentially form a hypersurface, and therefore must have measure zero. It should be noted that all known examples of positively curved manifolds can be obtained by this general approach. The method was pioneered by Eschenburg, who constructed the first inhomogeneous examples in 1982 [E1].

As a final remark on the Gromoll-Meyer sphere, we should mention the paper of Rigas $\mathrm{Ri}$. In this paper the author studies principal bundles over spheres, and some associated bundles. Using the theory of Riemannian submersions, he constructs bundles with non-negative sectional curvature. The Gromoll-Meyer sphere appears as the total space of the two of the bundles constructed, providing this sphere with two metrics of non-negative sectional curvature. However, it is not clear if these metrics are different from those discussed above, or indeed from the Grove-Ziller metrics described below. 
Grove and Ziller's paper [GZ1] represented a major advance in study of curvature on exotic spheres. They showed that in fact all exotic spheres in dimension seven which are $S^{3}$-bundles over $S^{4}$ (the Milnor spheres) admit metrics with non-negative sectional curvature. It should be noted that previously, Wilhelm Wi1 had shown the existence of a sequence of metrics $g_{i}$ on every Milnor sphere for which the diameter is bounded above by 1 , the sectional curvature is strictly positive at some point, and the lower bound for the sectional curavture is $-1 / i$. Like the GromollMeyer metric, these also admit an effective isometric $O(2) \times S O(3)$ action.

The Grove-Ziller results are achieved by studying manifolds of cohomogeneityone. A manifold of cohomogeneity-one admits a smooth action by a Lie group for which the space of orbits is one-dimensional. In the Riemannian case, where we insist that all actions are by isometries, the orbit space of a compact cohomogeneityone manifold can either be a circle (in which case all orbits are principal) or an interval (in which case there are two singular orbits). If all orbits are principal, it is easy to see that the manifold admits an invariant metric with non-negative curvature. The fundamental group of such a manifold is, however, infinite, so the manifold cannot admit a metric of positive sectional or positive Ricci curvature by the Myers Theorem. It is therefore the situation where the orbit space is an interval which Grove and Ziller study.

Topologically, a cohomogeneity-one manifold with two singular orbits is a union of two disk bundles. The base of each disk bundle is a singular orbit, and the boundary (along which they are glued to form the union) is a principal orbit. Note that the orbits, both principal and singular, are homogeneous spaces equipped with homogeneous metrics.

The Milnor spheres are associated (via the associated bundle construction) to certain $S O(4)$-principal bundles over $S^{4}$. These principal bundles admit cohomogeneity-one actions from $S O(4) \times S O(3)$ with codimension-two singular orbits. Grove and Ziller show that every cohomogeneity-one manifold with codimensiontwo singular orbits admits an invariant metric with non-negative sectional curvature. The Milnor spheres result now follows since each Milnor sphere can be described as the base of submersion (arising from the associated bundle construction) in which the total space has non-negative curvature. Grove and Ziller also investigate the symmetries of their exotic sphere metrics, and show that on each Milnor sphere there exists infinitely many inequivalent, isometric, almost-free actions of $S O(3)$.

Recently, Durán, Püttmann and Rigas DPR have described an explicit construction of all exotic spheres in dimension seven, which in some sense generalises the construction of the Gromoll-Meyer sphere. However, at the present time, no new curvature-related results have been obtained from this construction.

We now turn our attention to a different family of homotopy spheres: the Kervaire spheres. These spheres have dimension $4 n+1$, and can be defined as the boundary obtained by plumbing together two copies of the tangent disk bundle of $S^{2 n+1}$; see LM, p. 162]. Plumbing is a construction whereby disk bundles are glued together to create a new manifold with boundary. The basic idea is as follows. Consider two disc bundles, and for each bundle choose a locally trivial neighbourhood over a disc in the base. We glue the bundles by identifying these neighbourhoods. To do this we use a diffeomorphism that identifies the base disc of one neighbourhood with the fibre disc of the other, and vice versa. (Of course, this can only 
work if the dimensions match.) The resulting object is the so-called plumbing of the disc bundles, and can be made differentiable by simply straightening out the angles. For the basics of plumbing and applications to exotic sphere construction, see $[\mathrm{Br}, \mathrm{V} .2]$.

In some dimensions (e.g., 5, 13, 29, 61) the Kervaire sphere is diffeomorphic to the standard sphere; it is known that this can only happen in dimensions taking the form $2^{k}-3$ for $k \geq 3$, but the precise values of $k$ for which this happens are not known. This problem has become known as the "Kervaire invariant one problem"; see for example [La, Thm 5.6]. In the remaining dimensions the Kervaire sphere is exotic. The exotic Kervaire spheres are known to be the most symmetric of all exotic spheres $[\mathrm{HH}]$. In dimensions $4 n+1$, there are at most two homotopy spheres which bound a parallelisable manifold. The standard sphere is of course one of these. When it exists, the other sphere is a Kervaire sphere.

With a view towards giving a useful alternative description of Kervaire spheres, we next introduce the concept of a Brieskorn manifold.

Let $P\left(a_{0}, \ldots, a_{n+1}\right)$ be the complex polynomial

$$
P\left(a_{0}, \ldots, a_{n+1}\right)=z_{0}^{a_{0}}+\cdots+z_{n+1}^{a_{n+1}}
$$

and define the Brieskorn manifold $B\left(a_{0}, \ldots, a_{n+1}\right)$ to be the intersection of the zerolocus of $P\left(a_{0}, \ldots, a_{n+1}\right)$ with the unit sphere in $\mathbb{C}^{n+2}$. This is a smooth manifold of dimension $2 n+1$, which can be shown to be $(n-1)$-connected [M4, Thm 5.2].

For $n$ even and $d \equiv 3$ or $5 \bmod 8$, Brieskorn showed that the manifold $B(d, 2, \ldots, 2)$ is a Kervaire sphere $[\mathrm{Bk}$, and indeed every Kervaire sphere arises in this way. The Kervaire spheres admit a cohomogeneity-one action by $S O(n+1) \times S^{1}$. The singular orbits have codimension 2 and $2 n$. It is easy to see this action using the Brieskorn model: if $A \in S O(n+1)$ and $\theta \in S^{1}$ is viewed as the group of unit modulus complex numbers, then

$$
(A, \theta)\left(z_{0}, \ldots, z_{n+1}\right)=\left(\theta^{2} z_{0}, A\left(\theta^{d} z_{1}, \ldots, \theta^{d} z_{n+1}\right)\right) .
$$

In GVWZ, Grove, Verdiani, Wilking and Ziller show that the Kervaire spheres in all dimensions at least five admit no invariant metrics of non-negative sectional curvature for the cohomogeneity-one action. (This provides a counterexample to a conjecture of Grove and Ziller GZ1] which suggested that all compact cohomogeneity-one manifolds should admit an invariant metric of non-negative sectional curvature.) Note that it had been known since $1987[\mathrm{BH}]$ that Kervaire spheres of dimension at least nine do not admit $S O(n+1) \times S^{1}$ invariant cohomogeneity-one metrics of strictly positive sectional curvature.

Moving away from cohomogeneity-one actions, the following sphere theorem due to Berger and Klingenberg is well known (see for example $\mathrm{Au}$ or $\mathrm{S}$, for a general discussion, or E3] for a short direct proof). If $M^{n}$ is a compact simply connected Riemannian manifold for which the sectional curvature $K$ satisfies $\frac{1}{4}<K \leq 1$, then $M$ is homeomorphic to $S^{n}$. (Of course this does not exclude the possibility that $M$ is an exotic sphere.) Of more relevance to exotic spheres are differentiable analogues of this result: so-called differentiable sphere theorems. The idea here is that by a suitable choice of lower bound for $K$, we can force $M$ to be diffeomorphic to $S^{n}$. To the best of the authors' knowledge, the smallest lower bound found to date giving a differentiable sphere theorem is 0.654 by Suyama (see $[\mathrm{Su}]$ ). Of course this means that no exotic sphere can have a metric which is '0.654-pinched' in this way. It might be possible that some exotic sphere admits a quarter-pinched 
metric (that is, for which $\frac{1}{4}<K \leq 1$ ), though since we do not yet know if any exotic sphere admits positive sectional curvature, the answer to such a pinching question is probably quite distant! On the other hand, in We, Weiss shows that the Gromoll-Meyer sphere cannot support a quarter-pinched metric.

To conclude our survey of sectional curvature and exotic spheres, let us remark that there are other results that rule out the existence of positive sectional curvature metrics on exotic spheres in the presence of certain other geometric conditions. See for example GP] and GW] (especially Theorem C).

\section{RiCCi CURVATURE}

As a starting point for discussing the Ricci curvature of exotic spheres, let us recall a result of Lohkamp $[\mathrm{LO}$ according to which any manifold of dimension at least three admits a complete metric with (strictly) negative Ricci curvature. In particular this means that every exotic sphere admits such a metric.

Although the statement concerning negative Ricci curvature is straightforward, its proof is anything but that. In the case of positive Ricci curvature however, there is no easy existence statement. There are exotic spheres, starting in dimension 9, that do not admit any metric of positive Ricci curvature. This follows from a result of Hitchin [Hi] which asserts that these spheres in fact admit no metric of positive scalar curavture. For more detail see Section 4. The only known obstruction to positive Ricci curvature that is not an obstruction to positive scalar curvature comes from the classical theorem of Myers $\mathrm{My}$. This states that the fundamental group of a compact manifold with positive Ricci curvature must be finite. Of course for exotic spheres, the fundamental group cannot obstruct positive Ricci curvature.

For the sectional curvature, we have examples of exotic spheres with $K \geq 0$ and $K>0$ at some point, but no examples with $K>0$ everywhere. For the Ricci curvature, the equivalent situation could not arise. If a manifold admits a metric with non-negative Ricci curvature that is strictly positive at one point, then the metric can be deformed to one with positive Ricci curvature everywhere. This is a result of Ehrlich Eh]. (The result was first claimed by Aubin [Au, though there was an error in his proof.) In fact more can be said: if the original metric admits an isometric group action, it can be arranged that the deformed metric with positive Ricci curvature also admits an isometric action from the same group. (In the absence of such a point of Ricci positivity, there is no guarantee that the manifold can support a Ricci positive metric - even in the simply connected case. For example, a $K 3$ surface supports a Ricci flat metric but no metric of positive Ricci (or even positive scalar) curvature. It is unknown whether a simply connected manifold with a metric of non-negative Ricci and positive scalar curvature must always admit a Ricci positive metric.) All the results surveyed below concern the case of strictly positive Ricci curvature.

Historically, the first result concerning exotic spheres and positive Ricci curvature metrics was due to Cheeger [C]. He studied the Kervaire spheres, using the description as Brieskorn manifolds outlined in Section 2. Since these spheres admit a cohomogeneity-one action, topologically they split as a union of two disk bundles. Cheeger constructs a Ricci non-negative metric on each bundle isometric to a product near the boundary and invariant under the cohomogeneity-one action from $S O(n+1) \times S^{1}$. The union of the two bundles then acquires a Ricci non-negative metric. Cheeger observes that the Ricci curvature of the metric is strictly positive in some neighbourhood, so the result of Ehrlich mentioned above guarantees 
that the Ricci non-negative cohomogeneity-one metric can be deformed into a Ricci positive cohomogeneity-one metric.

Around the same time, Hernández-Andrade demonstrated in his thesis the existence of an infinite family of exotic spheres admitting Ricci positive metrics (see $[\mathrm{Hz})$. The results are obtained by viewing the exotic spheres in question as Brieskorn manifolds; however, the techniques involved differ from those of Cheeger.

The starting point is Brieskorn's paper [Bk] (see $[\mathrm{H}]$ for an English summary), which shows that every exotic sphere which bounds a parallelisable manifold can be described as a Brieskorn manifold (and not just the Kervaire spheres!). As noted in Section 2, with the possible exception of dimension four, the diffeomorphism classes of oriented homotopy spheres which bound a parallelisable manifold form a finite cyclic group under the connected sum operation. It was shown in $[\mathrm{KM}$ that in even dimensions, the only homotopy sphere bounding a parallelisable manifold is the standard sphere. In dimensions congruent to 1 modulo 4, we have the standard sphere and possibly a Kervaire sphere, as discussed in Section 2. The real interest from a curvature point of view, therefore, lay in dimensions congruent to 3 modulo 4. In these dimensions the number of exotic spheres bounding a parallelisable manifold grows more than exponentially with dimension. An explicit description of each of these spheres as a Brieskorn manifold can be given as follows. Brieskorn shows that in these dimensions the manifold $B(6 k-1,3,2, \ldots, 2)$ (as defined in Section 2) is a homotopy sphere bounding a parallelisable manifold for all $k \geq 1$, and in fact every such sphere can be realised as one of these Brieskorn manifolds for a suitable choice of $k$.

Hernández-Andrade's approach is to consider the metrics induced on Brieskorn manifolds from the ambient Euclidean metric. Since the Brieskorn manifolds can be described entirely by polynomial equations, one can write down relatively simple formulas for the Ricci curvature. Even so, these formulas are too complicated to allow easy detection of positive Ricci curvature. For this reason, Hernández-Andrade considers a deformation of the standard embedding. He replaces the zero-locus of $P$ by an explicit nearby variety diffeomorphic to the original. He also replaces the unit sphere in $\mathbb{C}^{n+1}$ by an explicit ellipsoid diffeomorphic to the sphere. The point is that the intersection between these two new manifolds is diffeomorphic to the original Brieskorn manifold, but the intersection is now Hermitian-orthogonal. It is this orthogonality that allows the curvature formulas to be simplified to a point where analysis is possible. The main result is that given integers $a_{0}, \ldots, a_{m} \geq 2$, there is some integer $N\left(a_{0}, \ldots, a_{m}\right)$ such that for $p \geq N$, the Brieskorn manifold $B\left(a_{0}, \ldots, a_{m}, 2, \ldots, 2\right)$ with $p$ copies of 2 after $a_{m}$ has a metric of positive Ricci curvature. The implication for exotic spheres is that in dimensions congruent to 3 modulo 4 the number of exotic spheres bounding a parallelisable manifold that admit a positive Ricci curvature metric grows without bound with the dimension. Note that this method is not sufficiently strong to show that every sphere bounding a parallelisable manifold in these dimensions admits a Ricci positive metric. For example the Gromoll-Meyer metric has positive Ricci curvature, but this cannot be established from Hernández-Andrade's approach.

A general approach to constructing Ricci positive metrics is to use bundle techniques. Given a compact fibre bundle with a Lie structure group, a metric for the base, a metric for the fibre for which the structure group acts by isometries, and a principal connection on the associated principal bundle, the construction of Vilms 
Vi shows that there is a canonical metric on the total space such that the projection to the base is a Riemannian submersion and the fibres are totally geodesic. It follows from the O'Neill formulas that if the base and fibre metrics have positive Ricci curvature, then there exists a Ricci positive metric on the total space of the bundle. This metric is obtained from the Vilms metric by uniformly shrinking fibres, that is, scaling the metric in fibre directions by a small constant $t$. The effect of this shrinking is to diminish the influence of the (principal) connection on the curvature. For the curvature formulas in this situation, see [B, 9.70].

It is an immediate corollary that all Milnor spheres admit metrics of positive Ricci curvature. It also follows that certain 15-dimensional exotic spheres, which can be expressed as 7-sphere bundles over the 8-sphere (see [Sh), admit positive Ricci curvature metrics. This was essentially the approach taken by Poor $[\mathrm{Po}]$ and Nash [Na who established the existence of Ricci positive metrics on these objects.

A further general approach to constructing Ricci positive manifolds is to use surgery. Surgery is an operation which alters the topology of a manifold in a precise way, and was introduced as a tool for use in problems relating to the classification of manifolds. A good introduction to the subject is $\underline{\mathbb{R}}$. We describe the basic idea. Begin with a manifold $M^{n+m}$ and a smooth embedding

$$
\iota: S^{n} \longrightarrow M^{n+m} \text {. }
$$

Assume the normal bundle of $\iota\left(S^{n}\right)$ is trivial. This means we can extend $\iota$ to an embedding

$$
e: S^{n} \times D^{m} \longrightarrow M
$$

Performing surgery involves cutting something out - the interior of the image of the embedding $e$-and stitching something in, namely a copy of $D^{n+1} \times S^{m-1}$. Note that the boundary of $S^{n} \times D^{m}$ equals $S^{n} \times S^{m-1}$, which agrees with the boundary of $D^{n+1} \times S^{m-1}$. This gluing-in is performed in the obvious manner determined by $e$. We say that the dimension of such a surgery is $n$, and the codimension is $m$. There is a subtlety with the above construction, however: In general the extended embedding $e$ is not unique. Viewing $S^{n} \times D^{m}$ as a bundle with base $S^{n}$ and fibre $D^{m}$, we can compose $e$ with a bundle isomorphism $S^{n} \times D^{m} \rightarrow S^{n} \times D^{m}$ to obtain a new embedding. In general, performing surgery using this new embedding will result in a manifold which is different from that obtained using $e$. Therefore, when performing surgery, it is imporant to specify the trivialisation of the normal bundle (that is, the choice of embedding $S^{n} \times D^{m} \rightarrow M^{n+m}$ ) being used.

The relevance of surgery to problems involving curvature was shown by Gromov and Lawson $\mathrm{GL}$, and Schoen and Yau $\underline{\mathrm{ScY}}$. Independently, they showed that the result of performing surgery of codimension at least three on a manifold with positive scalar curvature is again a manifold admitting a positive scalar curvature metric. This result has had profound implications for studying the existence of positive scalar curvature metrics, as we shall see in the last section.

Performing surgery within positive Ricci curvature is not quite so straightforward. The first major result in this direction was due to Sha and Yang [SY]. They assume that a tubular neighbourhood of the sphere on which surgery is to be performed is isometric to the product of a round metric on the sphere and a round normal disk. Using this isometry as the trivialisation of the normal bundle, they show that the manifold resulting from the surgery has positive Ricci curvature provided the dimension of the surgery is at least one, the codimension at least three, 
and the ratio of the radius of the sphere to the radius of the disk is sufficiently small. This result was developed further in Wr2, where is was showed that under the same metric assumptions, Ricci positivity can be preserved if different trivialisations of the normal bundle are used. This generalisation comes at the expense of tighter dimensional requirements.

This latter result was the key to proving that all exotic spheres which bound parallelisable manifolds admit Ricci positive metrics. This result appeared in Wr1, and settled the existence question that the work of Hernández-Andrade had gone some way to addressing. The result follows from the fact that all such exotic spheres can be constructed from the tangent sphere bundle of an even-dimensional sphere by a sequence of surgeries for which the surgery result in Wr2 applies. The appropriate sequence of surgeries can be identified by viewing the exotic spheres as the boundaries of plumbed manifolds. (See Section 2 for an outline of the plumbing construction for disc bundles.) If a disc bundle is plumbed with a disc bundle over a sphere, the effect of this on the boundary of the original bundle is precisely a surgery. Working with plumbed stably trivial disc bundles facilitates the construction of exotic spheres, as it is straightforward to detect when the boundary is an exotic sphere (and indeed which exotic sphere it is) in terms of topological invariants of the bounding manifold. (Note that the bounding manifold is parallelisable in the case that the disc bundles and base manifolds are all stably trivial.)

The results in Wr1 actually go further than this: every manifold arising as the boundary of a plumbing of disc bundles over spheres according to a simply connected graph admits a Ricci positive metric. This includes more exotic spheres than just those that bound parallelisable manifolds! The boundary of the manifold obtained by plumbing (the disc bundle of) the five-dimensional vector bundle over $S^{4}$ generating $K O\left(S^{4}\right)$ with (the disc bundle of) the non-trivial four-dimensional vector bundle over $S^{5}$, is an exotic sphere of dimension eight which does not bound a parallelisable manifold. However, by the above theorem it admits a metric of positive Ricci curvature. This is the only known example of an exotic sphere not bounding a parallelisable manifold that admits such a metric.

In Section 2 we discussed the work of Grove and Ziller on cohomogeneity-one metrics with non-negative sectional curavture GZ1. Following on from this result, they studied the existence of cohomogeneity-one metrics with positive Ricci curvature GZ2 They show that a compact manifold with a cohomogeneity-one action supports an invariant metric with positive Ricci curvature if and only if the fundamental group is finite. (Recall that the fundamental group of any compact manifold that admits a positive Ricci curvature metric must be finite, by the Myers Theorem [My.) Their approach could be viewed as a generalisation of Cheeger's metric construction for the Kervaire spheres discussed at the start of this section. This gives an alternative approach to the construction of Ricci positive metrics on certain exotic spheres, including all Milnor spheres. It is not clear whether this method generates Ricci positive metrics on any exotic spheres not previously known to admit them.

The most recent general approach to constructing Ricci positive metrics having applications to exotic spheres has its origin in the Calabi Conjecture [Ca]. This asserts that if $M$ is a compact Kähler manifold and $\rho$ is a real, closed (1,1)-form on $M$ with $[\rho]=2 \pi c_{1}(M)$, where $c_{1}(M)$ is the first (real) Chern class, then there exists a unique Kähler metric on $M$ with Kähler form cohomologous to the original 
Kähler form, for which the Ricci form is $\rho$. The conjecture was proved by Yau in $1978 \mathrm{Y}$. In the case where $c_{1}(M)>0$, in other words when $c_{1}(M)$ can be represented by a positive-definite real, closed $(1,1)$-form $\rho$, the conjecture implies that $M$ admits a metric of positive Ricci curvature. Although there is no known application of this result to the existence of positive Ricci curvature metrics on exotic spheres, there is an odd-dimensional analogue which has proved useful.

Sasakian geometry is special subfield of contact geometry. See [G], Et] or B] as a general reference. It can be viewed as an odd-dimensional analogue of Kähler geometry. A Riemannian manifold $(M, g)$ is called Sasakian if the holonomy group of the metric cone $\left(\mathbb{R}^{+} \times M, d r^{2}+r^{2} g\right)$ reduces to a subgroup of $U((n+1) / 2)$. In this case, the above cone is Kähler, and if $J$ is the complex structure, setting $\xi:=J(\partial / \partial r)$ defines a unit Killing vector field with the property that the sectional curvature of every plane containing $\xi$ is one. From a different perspective, $\xi$ can be viewed as the Reeb vector field of a contact structure on $M$ for which $g$ is an associated metric.

We should also mention the stronger concept of a 3-Sasakian manifold. Here, the holonomy group of the above metric cone reduces to a subgroup of $S p((n+1) / 4)$, and the cone itself is hyper-Kähler. Thus 3-Sasakian geometry is an odd-dimensional analogue of hyper-Kähler geometry. Note that the dimension of a 3-Sasakian manifold must be congruent to 3 modulo 4. A 3-Sasakian manifold has a triple of orthonormal Killing fields $\xi_{1}, \xi_{2}, \xi_{3}$ which give rise to a locally defined free isometric $S U(2)$ action on the manifold. Correspondingly, there exists a triple of contact forms on the manifold with $g$ as associated metric and $\xi_{1}, \xi_{2}, \xi_{3}$ as the Reeb vector fields. It is an open problem whether there exists 3-Sasakian metrics on exotic spheres. In particular, the only known 3-Sasakian metric on any homotopy sphere is the standard round metric. Every 3-Sasakian manifold is Einstein with positive scalar curvature. Informally, an Einstein metric has constant Ricci curvature. More precisely, the bilinear form associated to the Ricci curvature (the Ricci tensor) is proportional to the metric itself. See $[\mathrm{P}$, p. 31]. The scalar curvature of such a metric is equal to the dimension of the manifold multiplied by the constant of proportionality, so an Einstein metric with positive scalar curavture also has positive Ricci curvature.

In the context of Sasakian geometry, one has a result similar to the Calabi Conjecture (see BGN1, and also [EKA from which the result was developed). It is a consequence of this result that if an odd-dimensional manifold admits a positive Sasakian structure (see [BGN1]), then it must admit a metric of positive Ricci curvature. Boyer, Galicki and collaborators have exploited this to produce a very wide range of new examples of Ricci positive manifolds. Amongst these examples are exotic spheres [BGN2]. In particular they were able to construct new (Sasakian) metrics of positive Ricci curvature on all homotopy spheres which bound parallelisable manifolds, thus providing an alternative proof to the main result of Wr1. The exotic spheres are realised as Brieskorn manifolds, as described in $\S 2$ for dimensions $4 n+1$ and above for dimensions $4 n+3$.

Another ramification of this approach is that Boyer, Galicki and their collaborators have been able to determine the existence of Einstein metrics (actually Sasaki-Einstein) with positive scalar curvature on certain exotic spheres. The first examples of such Sasaki-Einstein metrics on exotic spheres were obtained in [BGK]. It was shown that all Kervaire spheres and all exotic spheres in dimension seven 
admit an Einstein metric with positive scalar curvature. In [BGKT] similar results were obtained for all exotic spheres in dimension 11, and also those in dimension 15 bounding a parallelisable manifold. (All exotic spheres in dimension 11 bound a parallelisable manifold.) The approach in these low dimensions is to perform a caseby-case check using a computer. It is conjectured that all exotic spheres bounding a parallelisable manifold admit an Einstein metric (indeed a Sasaki-Einstein metric) with positive scalar curvature BGK.

\section{SCAlar CURVAture}

The scalar curvature is the weakest of the three curvature notions we consider. Since the scalar curvature is the trace of the Ricci curvature form, the statements on positivity of the Ricci curvature presented in the last section already give some statements on positivity for the scalar curvature. In dimensions one and two there is no essential difference between the various curvature notions, so we will generally assume a manifold to have dimension at least three. A first good understanding of the nature of scalar curvature is arrived at through the classification result of Kazdan and Warner [K, Theorem 2.19] (proved in [KW1 and [KW2]). To state the result, we divide the collection of all smooth connected closed manifolds into three classes: those that have a Riemannian metric of non-negative scalar curvature which is positive at least at one point, those that have a Riemannian metric of nonnegative scalar curvature but do not belong to the first class, and the remaining ones. The result of Kazdan and Warner says that if $M$ is a smooth connected closed manifold of dimension at least three, then $M$ is in the first class if and only if every smooth function $f \in C^{\infty}(M)$ can be realized as the scalar curvature of an appropriate Riemannian metric on $M$. Furthermore, if $M$ is in the second class, then a smooth function $f \in C^{\infty}(M)$ is the scalar curvature of a Riemannian metric on $M$ if and only if $f(x)<0$ for some point $x \in M$ or $f \equiv 0$, while if $M$ belongs to the third class, then a smooth function $f \in C^{\infty}(M)$ can be realized as the scalar curvature of a Riemannian metric if and only if there is some point $x \in M$ with $f(x)<0$. Manifolds in the second class we call strongly scalar flat. To have some examples in mind, any closed manifold with a metric whose scalar curvature is positive everywhere is certainly contained in the first class. Thus spheres or suitable quotients of spheres (e.g., lens spaces) are contained in this class. A typical example for the second class is the torus: the existence of a flat metric shows that it belongs to one of the first two classes. However, Gromov and Lawson showed in GL that the torus does not admit a metric of positive scalar curvature, thus the torus is a scalar flat manifold. Probably the most mysterious class is the third one. In KW3, Theorem 5.1] Kazdan and Warner gave a sufficient, ad-hoc criterion for a manifold being contained in that class, which for example shows that the connected sum of $T^{4}$ and a $K 3$-surface is contained in it. For simply connected manifolds whose dimension is at least five, however, there is a completely satisfactory characterization of all three classes, due to results of Gromov and Lawson [GL], Stolz [St], and Futaki [F] (see below).

As a result of the enormous flexibility in arranging scalar curvature functions which follows from the Kazdan-Warner theorem, the only interesting question concerning positive scalar curvature issues on exotic spheres is to decide to which one of these classes they belong. We will see that there are no strongly scalar flat exotic spheres except possibly in dimensions three and four, and that for exotic 
spheres of dimension $\geq 5$ there is a computable invariant, the so-called $\alpha$-invariant, which effectively determines whether or not a given homotopy sphere possesses a Riemannian metric of positive scalar curvature.

The $\alpha$-invariant is a generalization of the so-called $\hat{A}$-genus and evolves from spin geometry. More precisely the $\alpha$-invariant of a closed spin manifold $M$ is the image of the spin bordism class of $M$ under the Atiyah-Bott-Shapiro homomorphism which maps the spin bordism ring to the coefficients of real $K$-theory. In the case of an exotic sphere, the invariant is trivial if the dimension of the exotic sphere is not equal to 1 or $2 \bmod 8$. In the remaining cases it is a $\mathbb{Z} / 2$-valued invariant which also can be defined completely geometrically. For simplicity assume that $M$ is a homotopy sphere of dimension $\geq 2$. In this case there is one and only one spin structure on $M$. If the dimension of $M$ is $1 \bmod 8$ the real spinor bundle $S$ comes equipped with a complex structure which is respected by the Dirac operator $D$ acting on the smooth sections on $S$. It follows that $\operatorname{Ker}(D)$ naturally has a complex structure. Similarly if the dimension of $M$ is $2 \bmod 8$, the real spinor bundle naturally has a quaternionic structure which is respected by the Dirac operator so that $\operatorname{Ker}(D)$ is a vector space over the quaterions $\mathbb{H}$ in natural way. The $\alpha$-invariant then can be computed as follows (compare [LM], §7):

$$
\alpha(M)= \begin{cases}\operatorname{dim}_{\mathbb{C}} K e r(D) \bmod 2 & \text { if } \operatorname{dim}(M) \equiv 1 \bmod 8, \\ \operatorname{dim}_{\mathbb{H}} K e r(D) \bmod 2 & \text { if } \operatorname{dim}(M) \equiv 2 \bmod 8 .\end{cases}
$$

The above interpretation of the $\alpha$-invariant can be regarded as an enhancement of the celebrated Atiyah-Singer index theorem. It is also the origin of its relationship to scalar curvature. In [Hi] Hitchin observed that the $\alpha$-invariant is an obstruction to the existence of a positive scalar curvature metric on $M$. His observation is based on a calculation of the Bochner-Weitzenböck curvature term for the spinor bundle on $M$ originally due to Lichnerowicz [Li] (however, see [LM], Theorem 8.8 for a more modern account). The latter yields the following expression of the Bochner-Weitzenböck formula for the square of the Dirac operator

$$
D^{2}=\nabla^{*} \nabla+\frac{1}{4} \text { scal, }
$$

where scal stands for the operator which multiplies by the scalar curvature function. Since the connection Laplacian $\nabla^{*} \nabla$ is a positive operator, it follows that $D^{2}$ is a strictly positive operator if scal $>0$. So is in this case one has $\operatorname{Ker}(D)=0$ and hence $\alpha(M)=0$.

One might ask whether there are exotic spheres which have a non-trivial $\alpha$ invariant. This question is answered in the affirmative by Adams and Milnor, who showed that the $\alpha$-invariant constitutes a surjective group homomorphism from the group $\theta_{n}$ of homotopy $n$-spheres (with the addition induced by the connected sum operation) onto the group $\mathbb{Z} / 2$, provided $n \equiv 1,2 \bmod 8$ and $n \geq 9$. Roughly speaking, Adams has shown [Ad, Theorem 1.2] that a non-trivial $\alpha$-invariant in dimensions $n \equiv 1,2 \bmod 8$ can always be realized through a stably framed closed manifold, while Milnor has shown [M3, Proof of Theorem 2] that one can alter an accordingly framed manifold to a homotopy sphere by a sequence of surgeries without changing its $\alpha$-invariant, provided $n \geq 9$ (and $n \equiv 1,2 \bmod 8)$.

The next natural question to ask is which exotic spheres with trivial $\alpha$-invariant do have Riemannian metrics of positive scalar curvature. For exotic spheres of dimension $\geq 5$, this question has been answered by Stolz in $\mathrm{St}$ ] where he shows that 
in fact any closed simply connected spin manifold of dimension $\geq 5$ with vanishing $\alpha$-invariant does have a Riemannian metric of positive scalar curvature. His results depend on a surgery result for scalar curvature which has been obtained independently by Gromov and Lawson in [GL] and Schoen and Yau in $[\mathrm{ScY}]$. The latter states that if a closed manifold $M$ of dimension $\geq 5$ is obtained by a sequence of surgeries of codimension $\geq 3$ from another closed manifold which has a Riemannian metric of positive scalar curvature, then $M$ also can be furnished with a positive scalar curvature metric. Gromov and Lawson already observed that the bordism result implies that any simply connected closed manifold of dimension at least five which is not spin has a metric of positive scalar curvature, the reason being that each such manifold can be obtained by a sequence of surgeries as above from a particular list of manifolds with standard metrics of positive scalar curvature. Homotopy spheres of dimension at least three, however, are spin manifolds. In the spin case the bordism result implies that a simply connected closed spin manifold $M$ of dimension at least five has a metric of positive scalar curvature if it is spin bordant to some closed spin manifold which has a metric of positive scalar curvature. Stolz then used methods from stable homotopy theory to show that in fact any closed simply connected spin manifold $M$ with $\alpha(M)=0$ is spin bordant to the total space of a bundle with the quaternionic projective plane $\mathbb{H}^{2}$ as fibre and structural group $I \operatorname{som}\left(\mathbb{H}^{2}\right)$, the group of isometries of $\mathbb{H}^{2}$. Given such a fibre bundle, one can use the O'Neill formulas to produce a metric of positive scalar curvature on the total space from the standard positive scalar curvature metric on $\mathbb{H}^{2}$ and an arbitrary Riemannian metric on the base. Note that the base of such a fibre bundle is compact, so one can squeeze the fibres so that at each point, the scalar curvature contribution coming from the fibre dominates the contribution coming from the base. Thus any $\mathbb{H}^{2}$-bundle of the above sort can be equipped with a metric of positive scalar curvature on its total space. Hence if $\alpha(M)=0$, the surgery result of Gromov and Lawson, respectively Schoen and Yau, can be used to construct a metric of positive scalar curvature on $M$ from a positive scalar curvature metric on a spin bordant $\mathbb{H}^{2}$-bundle. These bordism results give a good description of those simply connected manifolds of dimension at least five which belong to the first class in the Kazdan-Warner classifaction; namely, such a manifold belongs to this class unless it is spin and has a non-trivial $\alpha$-invariant.

If the $\alpha$-invariant of an exotic sphere $M$ is non-trivial, it remains to ask whether or not $M$ is strongly scalar flat. We know that in dimensions one and two there are no exotic spheres. On the other hand one knows that there are no scalar flat exotic spheres in dimensions $\geq 5$. This is a simple consequence of the main theorem of Futaki in $[\mathrm{F}$. Futaki showed that a closed simply connected manifold $M$ of dimension $\geq 5$ is strongly scalar flat if and only if $\alpha(M) \neq 0$ and $M=$ $M_{1} \times \cdots \times M_{r}$, where each factor is a Ricci-flat Kähler manifold or a Riemannian manifold with $\operatorname{Spin}(7)$-holonomy. This statement in a way also follows from the Bochner-Weitzenböck formula. If $\alpha(M) \neq 0$, one has $\operatorname{Ker}(D) \neq 0$. Thus if $M$ is equipped with a scalar flat metric, this implies that $M$ has a parallel spinor, and the latter by a result of Hitchin $[\mathrm{H}]$ yields that $M$ must be Ricci-flat and must have special holonomy (compare $[\mathrm{F}$. on page 28). Note that a compact Kähler manifold has a non-trivial second cohomology group, while a Riemannian manifold with $\operatorname{Spin}(7)$-holonomy has dimension eight. Since every homotopy sphere in particular is a homology sphere, a homotopy sphere of dimension $\geq 5$ with a non-vanishing 
$\alpha$-invariant cannot have the same cohomology as a product of the above form. In particular, homotopy spheres of dimension $\geq 5$ with a non-vanishing $\alpha$-invariant are not strongly scalar flat. The last statement can also be found in D, Proposition 1.1] where Dessai derives it from another implication of Futaki's result.

In the remaining dimensions three and four, the scalar curvature classification of homotopy/exotic spheres depends on various versions of the Poincaré conjecture. As already mentioned in the Introduction, Perelman recently proved the classical Poincaré conjecture in dimension three, thus there are no homotopy 3 -spheres apart from the standard 3-sphere. On the other hand, the smooth Poincaré conjecture in dimension four which claims that there are no exotic 4 -spheres has yet to be resolved. Many people believe that it is not true since there are many 4-manifolds which have non-isomorphic differentiable structures. However, to the best of the authors' knowledge, there is not even a candidate for a counterexample at the present time. In particular, there is nothing to be said concerning special metrics on exotic 4-spheres.

\section{ABout THE AUTHORS}

Michael Joachim is a Senior Lecturer at Westfälische Wilhelms-Universität in Münster, Germany.

David Wraith is a Senior Lecturer at the National University of Ireland in Maynooth.

\section{REFERENCES}

[Au T. Aubin, Métriques riemanniennes et courbure, J. Differential Geometry 4 (1970), 383-424. MR0279731(43:5452)

[Ad] J.F. Adams, On the groups $J(X)$. IV, Topology 5 (1966), 21-71. MR0198470 (33:6628)

[B] A.L. Besse, Einstein Manifolds, Springer-Verlag, Berlin (2002). MR867684 (88f:53087)

[BGK] C.P. Boyer, K. Galicki, J. Kollár, Einstein metrics on spheres, Ann. of Math. (2) 162 no. 1 (2005), 557-580. MR2178969 (2006j:53058)

[BGKT] C.P. Boyer, K. Galicki, J. Kollár, E. Thomas, Einstein metrics on exotic spheres in dimensions 7, 11 and 15, Experiment. Math. 14 no. 1 (2005), 59-64. MR.2146519 (2006a:53042)

[BGN1] C.P. Boyer, K. Galicki, M. Nakamaye, On positive Sasakian geometry, Geom. Dedicata 101 (2003), 93-102. MR.2017897 (2005a:53072)

[BGN2] C.P. Boyer, K. Galicki, M. Nakamaye, Sasakian geometry, homotopy spheres and positive Ricci curvature, Topology 42 (2003), 981-1002. MR.1978045 (2004c:53055)

[BH] A. Back, W.Y. Hsiang, Equivariant geometry and Kervaire spheres, Trans. Amer. Math. Soc. 304 (1987), 207-227. MR.906813 (88m:53073)

[Bk] E. Brieskorn, Beispiele zur Differentialtopologie von Singularitäten, Invent. Math. 2 (1966), 1-14. MR.0206972 (34:6788)

[Bl] D.E. Blair, Riemannian geometry of contact and symplectic manifolds, Progress in Mathematics, 203. Birkhäuser Boston, Inc., Boston, MA, 2002. MR.1874240 (2002m:53120)

[Br] W. Browder, Surgery on simply-connected manifolds, Springer, Berlin, (1972). MR0358813 (50:11272)

$[\mathrm{Bu}]$ D. Burago, Y. Burago, S. Ivanov, A course in metric geometry, Graduate Studies in Mathematics, 33, American Mathematical Society, Providence, RI, 2001. MR 1835418 (2002e:53053)

[C] J. Cheeger, Some examples of manifolds of nonnegative curvature, J. Differential Geometry, 8 (1973), 623-628. MR.0341334 (49:6085)

[Ca] E. Calabi, On Kähler manifolds with vanishing canonical class, Algebraic Geometry and Topology. A symposium in honor of S. Lefschetz, Princeton Univ. Press, Princeton, N.J., 1957, pp. 78-89. MR0085583(19:62b) 
[Ce] J. Cerf, Sur les difféomorphismes de la sphère de dimension trois $\left(\Gamma_{4}=0\right)$, Lecture Notes in Mathematics, 53, Springer-Verlag, Berlin-New York, 1968. MR0229250 $(37: 4824)$

[Ch] I. Chavel, Riemannian geometry. A modern introduction, Cambridge Tracts in Mathematics, 108, Cambridge Univ. Press, 1993. MR1271141 (95j:53001)

[D] A. Dessai, On the topology of scalar-flat manifolds, Bull. London Math. Soc. 33 (2001), 203-209. MR1815425 (2002b:53063)

[doC] M. do Carmo, Riemannian geometry, Birkhäuser Boston, Inc., Boston, MA, 1992. MR:1138207 (92i:53001)

[DPR] C. Durán, T Püttmann, A. Rigas, An infinite family of Gromoll-Meyer spheres, arXiv:math.DG/0610349.

[Du] C. Durán, Pointed Wiedersehen metrics on exotic spheres and diffeomorphisms of $S^{6}$, Geom. Dedicata 88 (2001), 199-210. MR.1877216 (2002i:57044)

[E1] J.-H. Eschenburg, New examples of manifolds with strictly positive curvature, Invent. Math. 66 (1982) no. 3, 469-480. MR662603 (83i:53061)

[E2] J.-H. Eschenburg, Freie isometrischen Aktionen auf kompakten Lie-Gruppen mit positiv gekrümmten Orbiträumen, Schriftenreihe Math. Inst. Univ. Münster 32 (1984). MR758252(86a:53045)

[E3] J.-H. Eschenburg, Local convexity and nonnegative curvature-Gromov's proof of the sphere theorem, Invent. Math. 84 (1986), 507-522. MR837525(87j:53080)

[E4] J.-H. Eschenburg, Cohomology of biquotients, Manuscripta Math. 75 (1992) no. 2, 151166. MR1160094 (93e:57070)

[E5] J.-H. Eschenburg, Almost positive curvature on the Gromoll-Meyer 7-sphere, Proc. Amer. Math. Soc. 130 no. 4, (2002), 1165-1167. MR1873792 (2002i:53045)

[Eh] P.E. Ehrlich, Metric deformations of curvature, I. Local convex deformations, Geom. Dedicata 5 (1976), 1-23. MR0487886 (58:7482a)

[EK] J.-H. Eschenburg, M. Kerin, Almost positive curvature on the Gromoll-Meyer 7-sphere, Proc. Amer. Math. Soc. posted on April 23, 2008, PII S0002-9939(08)09429-X (to appear in print).

[EKA] A. El Kacimi-Alaoui, Opérateurs transversalement elliptiques sur un feuilletage riemannien et applications, Composito Math. 73 (1990), no. 1, 57-106. MR.1042454(91f:58089)

[Et] J. Etnyre, Introductory lectures on contact geometry, Proc. Sympos. Pure Math. 71 (2003), 81-107, Amer. Math. Soc., Providence, RI, 2003. MR2024631 (2005b:53139)

[F] A. Futaki, Scalar-flat closed manifolds not admitting positive scalar curvature metrics, Invent. Math. 112 (1993), 23-29. MR1207476 (94f:53072)

[Fr] M. Freedman, The topology of four dimensional manifolds, J. Differential Geom. 17 (1982) no. 3, 357-453. MR679066 (84b:57006)

[FQ] M. Freedman and F. Quinn, Topology of 4-manifolds, Princeton Mathematical Series 39, Princeton University Press, Princeton, N.J., 1990. MR1201584 (94b:57021)

[G] H. Geiges, Contact geometry, Chapter 5 of the Handbook of Differential Geometry, Volume 2, Elsevier/North-Holland, Amsterdam, 2006. MR2194671 (2007c:53123)

[GL] M. Gromov, H.B. Lawson, The classification of simply connected manifolds of positive scalar curvature, Ann. of Math. (2) 111 (1980), 423-434. MR.577131(81h:53036)

[GM] D. Gromoll, W.T. Meyer, An exotic sphere with nonnegative sectional curvature, Ann. of Math. (2) 100 (1974), 401-406. MR0375151 (51:11347)

[GP] K. Grove, P. Petersen, A pinching theorem for homotopy spheres, J. Amer. Math. Soc. 3 no.3, (1990), 671-677. MR.1049696 (91e:53040)

[GVWZ] K. Grove, L. Verdiani, B. Wilking, W. Ziller, Non-negative curvature obstructions in cohomogeneity one and the Kervaire spheres, Ann. Sc. Norm. Super. Pisa Cl. Sci. (5) 5 (2006), no. 2, 159-170. MR2244696 (2007h:53051)

[GW] K. Grove, F. Wilhelm, Metric constraints on exotic spheres via Alexandrov geometry, J. Reine Angew. Math. 487 (1997) 201-217. MR1454266 (98d:53060)

[GZ1] K. Grove, W. Ziller, Curvature and symmetry of Milnor spheres, Ann. of Math. (2) 152 (2000), 331-367. MR 1792298 (2001i:53047)

[GZ2] K. Grove, W. Ziller, Cohomogeneity one manifolds with positive Ricci curvature, Invent. Math. 149 (2002), 619-646. MR.1923478 (2004b:53052)

[H] F. Hirzebruch, Singularities and exotic spheres, Séminaire Bourbaki, 1966/67, Exp. 314, Textes des conférences, o.S., Paris: Institut Henri Poincaré 1967. 
[HBJ] F. Hirzebruch, T. Berger, R. Jung, Manifolds and modular forms, Aspects of Mathematics, E20. Friedr. Vieweg \& Sohn, Braunschweig, 1992. MR1189136 (94d:57001)

[HH] W.-C. Hsiang, W.-Y. Hsiang, The degree of symmetry of homotopy spheres, Ann. of Math. (2) 89 (1969), 52-67. MR0239621 (39:978)

[Hi] N. Hitchin, Harmonic Spinors, Adv. in Math. 14 (1974), 1-55. MR0358873 (50:11332)

[HM] M. Hirsch, B. Mazur, Smoothings of piecewise linear manifolds, Annals of Mathematical Studies 80, Princeton Univ. Press, Princeton, NJ, 1974. MR0415630 (54:3711)

[Hz] H. Hernández-Andrade, A class of compact manifolds with positive Ricci curvature, Proc. Sympos. Pure Math. Vol. 27, 73-87, Amer. Math. Soc., Providence, RI, 1975. MR0380668 (52:1565)

[K] J.L. Kazdan, Prescribing the curvature of a Riemannian manifold, CBMS Reg. Conf. Ser. Math., 57, Amer. Math. Soc., Providence, RI, 1985. MR787227 (86h:53001)

[KM] M. Kervaire, J. Milnor, Groups of homotopy spheres. I. Ann. of Math. (2) 77 (1963), 504-537. MR0148075 (26:5584)

[KS] R. Kirby, L. Siebenmann, Foundational essays on topological manifolds, smoothings, and triangulations, Annals of Mathematical Studies, No. 88, Princeton Univ. Press, Princeton, NJ, 1977. MR0645390 (58:31082)

[KW1] J.L. Kazdan, F.W. Warner, Existence and conformal deformation of metrics with prescribed Gaussian and scalar curvatures, Ann. of Math. 101 (1975), 317-331. MR0375153 $(51: 11349)$

[KW2] J.L. Kazdan, F.W. Warner, A direct approach to the determination of Gaussian and scalar curvature functions, Invent. Math. 28 (1975), 227-230. MR0375154 (51:11350)

[KW3] J.L. Kazdan, F.W. Warner, Prescribing curvatures, Differential geometry, Proc. Sympos. Pure Math., Vol. XXVII Part 2, 309-319, Amer. Math. Soc., Providence, RI, 1975. MR0394505 (52:15306)

[KZ] V. Kapovitch, W. Ziller, Biquotients with singly generated rational cohomology, Geom. Dedicata 104 (2004), 149-160. MR2043959 (2005e:22014)

[La] T. Lance, Differentiable structures on manifolds, Surveys on Surgery Theory, Vol. 1, 73-104, Annals of Mathematical Studies, 145, Princeton Univ. Press, Princeton, NJ, 2000. MR1747531 (2001d:57035)

[LM] H.B. Lawson, M.-L. Michelsohn, Spin geometry, Princeton Math. Series, 38, Princeton University Press, Princeton, NJ, 1989. MR1031992 (91g:53001)

[Li] A. Lichnerowicz, Spineurs harmoniques, C. R. Acad. Sci. Paris 257 (1963), 7-9. MR0156292 (27:6218)

[Lo] J. Lohkamp, Metrics of negative Ricci curvature, Ann. of Math. (2) 140 (1994), 655-683. MR1307899 (95i:53042)

[M1] J. Milnor, On manifolds homeomorphic to the 7-sphere, Ann. of Math. (2) 64 (1956), 399-405. MR0082103(18:498d)

[M2] J. Milnor, Morse Theory, Annals of Mathematical Studies, 51, Princeton Univ. Press, Princeton, NJ, 1963. MR0163331 (29:634)

[M3] J. Milnor, Remarks concerning spin manifolds, Differential and Combinatorial Topology, 55-62, Princeton Univ. Press, Princeton, NJ, 1965. MR0180978 (31:5208)

[M4] J. Milnor, Singular points of complex hypersurfaces, Annals of Mathematical Studies, 61, Princeton Univ. Press, Princeton, NJ, 1968. MR0239612 (39:969)

[M5] J. Milnor, Classification of $(n-1)$-connected $2 n$-dimensional manifolds and the discovery of exotic spheres, Surveys on Surgery Theory, Vol. 1, 25-30, Annals of Mathematical Studies 145, Princeton Univ. Press, Princeton, NJ, 2000. MR.1747528(2001c:57002)

[MS] J. Milnor, J. Stasheff, Characteristic Classes, Annals of Mathematical Studies, 76, Princeton Univ. Press, Princeton, NJ, 1974. MR0440554 (55:13428)

[My] S.B. Myers, Riemannian manifolds with positive mean curvature, Duke Math. J. 8 (1941), 401-404. MR0004518 (3:18f)

[Na] J. Nash, Positive Ricci curvature on fiber bundles, J. Differential Geom. 14 (1979), 241-254. MR587552 (81k:53039)

[ON] B. O'Neill, The fundamental equations of a submersion, Michigan Math. J. 13 (1966), 459-469. MR0200865 (34:751)

[P] P. Petersen, Riemannian Geometry, Graduate Texts in Mathematics, 171, SpringerVerlag, (1998). MR1480173 (98m:53001) 
[P1] G. Perelman, The entropy formula for the Ricci flow and its geometric applications, http://arxiv.org/math.DG/0211159

[P2] G. Perelman, Ricci flow with surgery on three-manifolds, http://arxiv.org/ math.DG/0303109

[P3] G. Perelman, Finite extinction time for the solutions to the Ricci flow on certain threemanifolds, http://arxiv.org/math.DG/0307245

[Po] W.A. Poor, Some exotic spheres with positive Ricci curvature, Math. Ann. 216 (1975), 245-252. MR0400110 (53:3945)

[PW] P. Petersen, F. Wilhelm, An exotic sphere with positive sectional curvature, arXiv: 0805.0812v1 [math.DG]

[R] A. Ranicki, Algebraic and Geometric Surgery, Oxford Mathematical Monographs, Oxford University Press, (2002). MR.2061749 (2005e:57075)

[Ri] A. Rigas, Some bundles of nonnegative curvature, Math. Ann. 232 (1978), 187-193. MR0464254 (57:4188)

[S] K. Shiohama, Recent developments in sphere theorems, Differential geometry. Proc. Sympos. Pure Math., 551-576, Vol. 54, Part 3, Amer. Math. Soc., Providence, RI, 1993. MR:1216646 (94d:53071)

[Si] W. Singhof, On the topology of double coset manifolds, Math. Ann. 297 (1993) no. 1, 133-146. MR.1238411 (94k:57054)

[ScY] R. Schoen, S.T. Yau, On the structure of manifolds of positive scalar curvature, Manuscr. Math. 28 (1979), 159-183. MR535700 (80k:53064)

[Sh] N. Shimada, Differentiable structures on the 15-sphere and Pontrjagin classes of certain manifolds, Nagoya Math. J. 12 (1957), 59-69. MR0096223(20:2715)

[Sm1] S. Smale, Generalized Poincaré's conjecture in dimensions greater than four, Ann. of Math. (2) 74 (1961), 391-406. MR0137124 (25:580)

[Sm2] S. Smale, On the structure of manifolds, Amer. J. Math. 84 (1962), 387-399. MR0153022 $(27: 2991)$

[St] S. Stolz, Simply connected manifolds with positive scalar curvature, Ann. of Math. (2) 136 (1992), 511-540. MR.1189863 (93i:57033)

[SY] J.P. Sha, D.G. Yang, Positive Ricci curvature on the connected sums of $S^{n} \times S^{m}$, J. Differential Geometry 33 (1991), 127-138.

[Su] Y. Suyama, A differentiable sphere theorem by curvature pinching. II, Tohoku Math. J. (2) 47 no. 1 (1995), 15-29. MR 1311439 (95k:53048)

[To] B. Totaro, Cheeger manifolds and the classification of biquotients, J. Differential Geom. 61 (2002), 397-452. MR1979366 (2004b:53075)

[Vi] J. Vilms, Totally geodesic maps, J. Differential Geom. 4 (1970), 73-79. MR0262984 $(41: 7589)$

[We] M. Weiss, Pinching and concordance theory, J. Differential Geom. 38 (1993), 387-416. MR.1237489 (95a:53057)

[Wi1] F. Wilhelm, Exotic spheres with lots of positive curvatures, J. Geom. Anal. 11 (2001), 163-188. MR:1829354 (2002c:53062)

[Wi2] F. Wilhelm, An exotic sphere with positive curvature almost everywhere, J. Geom. Anal. 11 (2001), 519-560. MR1857856 (2002f:53056)

[Wr1] D. Wraith, Exotic spheres with positive Ricci curvature, J. Differential Geom. 45 (1997), 638-649. MR1472892 (98i:53058)

[Wr2] D. Wraith, Surgery on Ricci positive manifolds, J. Reine Angew. Math. 501 (1998), 99-113. MR 1637825 (99j:53050)

[Y] S.T. Yau, On the Ricci curvature of a compact Kähler manifold and the complex Monge-Ampere equation. I, Comm. Pure Appl. Math. 31 (1978), 339-411. MR480350 (81d:53045)

Mathematisches Institut, Westfälische Wilhelms-Universität Münster, Einsteinstr. 62, 48149 MÜnster, Germany

E-mail address: joachim@math.uni-muenster.de

Department of Mathematics, national University of Ireland Maynooth, Maynooth, Co. Kildare, Ireland

E-mail address: David.Wraith@nuim.ie 